\title{
Análise crítica dos livros didáticos de matemática de Moçambique à luz da Teoria Antropológico do Didático
}

Critical analysis of mozambique's mathematical books in the light of the anthropological theory of didathic

\author{
Geraldo Vernijo Deixa ${ }^{1}$ \\ Rosalino Subtil Chicote ${ }^{2}$ \\ Laurindo Sobra ${ }^{3}$
}

\section{Resumo}

Este artigo apresenta os resultados da pesquisa que analisa como o ensino da trigonometria é proposto nos livros didáticos de Matemática de Moçambique. Para o efeito, foi analisado o capítulo de trigonometria em quatro livros didáticos do Ensino Secundário Geral. A pesquisa é de carácter qualitativo com enfoque em análise documental. Para a análise de dados, recorreu-se a Análise de Conteúdo (BARDIN, 2012). Da análise das tarefas presentes nesses livros constatamos o predomínio de tarefas do gênero, de cálculo com uso de propriedades, sendo que 80 das 123 tarefas estão ligadas a este gênero. Aferimos ainda que 26 das 123 exigem habilidades de manipulação dos registros e, 17 das 123 tarefas exigem a leitura e interpretação. Esses resultados nos levam à conclusão de que os livros analisados não cumprem as sugestões dos programas de Ensino de Matemática. Abre-se, deste modo, uma oportunidade para investigar as percepções dos professores de matemática de Moçambique sobre as diferentes classificações das tarefas.

Palavras-chave: Trigonometria; Tarefa; Livro didáctico de Matemática.

\section{Abstract}

This article presents the results of a research that analyzes how the teaching of trigonometry is proposed in the didactic books of Mathematics of Mozambique. For that purpose, the chapter on trigonometry was analyzed in four didactic books of General Secondary Education. The research is qualitative with a focus on documentary research. For the analysis of data, we used the Content Analysis (BARDIN, 2012). From the analysis of the tasks present in these books we found that there is a predominance of gender tasks, to calculate with use of properties, and 80 of the 123 tasks are linked to this gender. We also note that 26 out of 123 require record manipulation skills and 17 of the 123 tasks require reading and interpretation. These results lead us to the conclusion that the books analyzed do not fulfill the suggestions of the Mathematics Teaching programs. This opens an opportunity to investigate the perceptions of teachers of mathematics in Mozambique about the different classifications of tasks.

Keywords: Trigonometry; Assignment; Mathematics Teaching Book

\footnotetext{
1 Universidade Licungo, Moçambique | gdeixa@gmail.com

2 Universidade Rovuma, Moçambique | rschicote1@gmail.com

3 Universidade Licungo, Moçambique |laurindosobra@gmail.com
} 


\section{Considerações iniciais}

A trigonometria tem sido um dos conteúdos mais problemático no ensino moçambicano. Há relatos frequentes de alunos segundo os quais quando se chega a esse capítulo, os professores indicam-no como trabalho de investigação em que os alunos vão realizando de forma independente. Muitas vezes, esses trabalhos são arquivados nas escolas e quase não se discute esse conteúdo matemático.

Nos livros didáticos da $10^{\mathrm{a}}$ e $11^{\mathrm{a}}$ classe, esse conteúdo matemático é lecionado no $3^{\circ}$ trimestre. Na 10a classe (para alunos de 14 e 15 anos de idade) o mesmo conteúdo é lecionado em seis semanas correspondentes a 24 aulas. Já na $11^{1 a}$ classe (para alunos de $15 \mathrm{a}$ 16 anos de idade) é lecionado em treze semanas correspondentes a 26 aulas. Na $12^{a}$ classe, não há uma indicação clara do trimestre nem a quantidade de aulas a serem lecionadas visto que está inserido nas funções reais de variável real, portanto, é tratado como revisão.

O Sistema Nacional de Educação moçambicana (SNE) divide-se em três trimestres letivos. Tanto nos programas quanto nos livros didáticos, a trigonometria é tratada no último trimestre, nesse período, muitas vezes, já não sobra tempo para aprofundamento desse conteúdo matemático. Estão por detrás desse incumprimento, excessos de feriados, condições climáticas de cada região que dita o início tardio das aulas, o excesso de alunos por turma que muitas vezes dificulta o acompanhamento do ritmo de aprendizagem dos mesmos, as visitas dos dirigentes do governo que, muitas vezes, obrigam os alunos a fazer parte dos comícios e de outros eventos afins.

Um outro elemento, não menos importante, que concorre para incumprimento dos Programas de Ensino pode ser o problema docente. O professor gasta muito tempo trabalhando em tarefas de consolidação, os chamados exercícios. Poucas vezes, trabalha nas tarefas de produção (resolução de problemas). Neste sentido, tem havido problemas em distinguir o que é essencial num determinado capítulo e como trabalhá-lo.

De acordo com Santos e Freitas (2017, p. 53), "a matemática realizada em sala de aulas em torno de um tema estudado pode ser analisada por meio da Organização Matemática". Nesse sentido, analisamos o ensino da trigonometria por meio dos livros didáticos moçambicanos. Essa análise foi feita com base na Teoria Antropológica do Didático (TAD). Conforme apontam Santos e Freitas (2017) para TAD tudo é objeto. O uso desta teoria justifica-se por ser uma das ferramentas que muitas pesquisas em Educação Matemática recorrem para analisar literatura didática na área de Educação Matemática. Esta teoria é de origem francesa, proposta por Chevallard, em 1999.

Tal como em outros países do mundo, em Moçambique, o livro didático (o livro do aluno), é fortemente utilizado pelo professor para preparar e planejar suas aulas, ajustar tarefas para os alunos, elaborar testes, entre outras aplicações. Segundo Bittar (2017, p. 365) "o Livro Didático é o principal material utilizado pelo professor no preparo das suas aulas. Muitas vezes este tem sido quase exclusivamente o único material que o professor usa".

São poucas alternativas que o professor moçambicano tem para escolher esse material para o uso em sala de aulas visto que são poucas as possibilidades oferecidas pelas 
editoras. Por reconhecer a importância que o livro didático tem para o ensino e para aprendizagem em matemática, analisamos como os conteúdos matemáticos são propostos e que ênfase é dada a capítulo para poder auxiliar o professor a melhorar o modo como os comunicar aos alunos. Também reconhecemos que o modo como o professor ensina está fortemente ligado à maneira como os conteúdos são abordados em livros didáticos.

Neste artigo, a nossa intenção é a apresentação dos resultados da pesquisa derivados da seguinte questão: como é proposto o ensino de Trigonometria no Ensino Secundário Geral moçambicano?

O livro como material didático é necessário para os processos de ensino e de aprendizagem tanto para o professor quanto para o aluno. Porém, nem sempre devemos confiar num único livro para o ensino de certo conteúdo tendo em conta que tal material pode apresentar erros na sua abordagem; tarefas que não se adequam a realidade cognitiva e local do aluno; excesso de exercícios de memorização; inexistência de contextualizações; inadequação das articulações entre abordagens de conceitos; deficiências dos recursos visuais mobilizados e metodologias antiquadas e incompatíveis com a classe e com a idade dos alunos.

Com base na exposição anterior, pode-se percebe a necessidade de usar mais de um livro didático como forma de minorar os possíveis erros que eventualmente tenham ocorrido no processo de elaboração dos livros aprovados pelo Ministério de Educação e Desenvolvimento Humano. Assim, o uso de várias fontes de consulta para a preparação e o planejamento de aulas pode ampliar a visão do professor relativamente aos conteúdos expostos, abrindo possíveis acomodações da realidade do aluno. A diversificação de fontes de consulta permite ainda a aquisição de vários pontos de vista sobre o mesmo objeto matemático. Essa multiplicidade de visões concede ao aluno maior capacidade de comunicação, de raciocínio bem como de descrição a respeito do objeto matemático em estudo.

Nessa direção, o Programa de Matemática do Ensino Secundário moçambicano indica que a aprendizagem da Matemática visa desenvolver,

O raciocínio lógico ao operar com conceitos e procedimentos, usando métodos apropriados; a capacidade de comunicar, ao enunciar propriedades e definições, assim como ao transcrever mensagens matemáticas da linguagem simbólica (fórmulas, símbolos, tabelas, gráficos) para a linguagem corrente e vice-versa; habilidades tais como: classificar, seriar, relacionar, reunir, representar, analisar, sintetizar, deduzir, provar e julgar (INDE, p. 43).

O desenvolvimento dessas habilidades exige da parte do professor uma criatividade. Também os livros didáticos de Matemática devem conter tarefas que possam conduzir alunos a desenvolverem as habilidades descritas acima. No entanto, o uso de único livro pode confundir o professor e pode ainda provocar dificuldades na aquisição dessas capacidades pelos alunos. Pode ainda não abrir espaço para o desenvolvimento de certas 
habilidades como por exemplo a matematização. Pois, geralmente, há excesso de tarefas que são classificadas como exercícios. Estas exigem um baixo nível de exigência cognitiva no seio do aluno.

O uso de vários livros para o preparo de aulas permite que o professor se reflicta que,

(...) A tarefa pode surgir de diversas maneiras: pode ser formulada pelo professor e proposta ao aluno, ser da iniciativa do próprio aluno e resultar até de uma negociação entre o professor e o aluno. Além disso, a tarefa pode ser enunciada explicitamente logo no início do trabalho ou ir sendo constituída de modo implícito à medida que este vai decorrendo. É formulando tarefas adequadas que o professor pode suscitar a actividade (PONTE, 2005, p. 11).

Esse entendimento pode abrir caminho para que um professor compreenda a relevância de prever tipologias de tarefas para aquisição de diferentes habilidades. Ponte (2005) classifica as tarefas de acordo com o seu grau de desafio em problema, exercício, investigação, projeto e tarefas de modelação. Sendo que, um exercício é uma tarefa fechada e de desafio reduzido; um problema é uma tarefa também fechada, mas com elevado desafio; uma investigação tem um grau de desafio elevado, mas é uma tarefa aberta.

A capacidade de comunicar é destacada ainda quando o Programa de Matemática da $12^{a}$ classe indica que o aluno ao terminar a $12^{a}$ classe deve ser capaz de " interpretar fenómenos e resolver problemas recorrendo a funções e seus gráficos e interpretar e utilizar representações matemáticas (tabelas, gráficos, expressões e símbolos) " (INDE, 2010, p. 9).

O Programa de Ensino da Matemática (PEM) da 10ª classe sugere que o tratamento do conteúdo da trigonometria seja feito observando a origem etimológica do termo e, em seguida, por meio de situações - problemas que reflitam o cotidiano do aluno. O mesmo documento destaca que no estudo desse conteúdo matemático se dê maior tempo ao tratamento dos conceitos e às aplicações ligadas à problemas reais. Nesse sentido, sugerese a redução da ênfase em exercícios de mero cálculo, ou seja, os de memorização. O recurso às situações - problema requer que o professor esteja preparado cientificamente e didaticamente, daí que o PEM destaca que o sucesso para o tratamento da trigonometria em sala de aula passa necessariamente pelo reconhecimento da importância do ensino desta que deve ficar claro primeiro para o professor e depois para o aluno (INDE, 2010, p.45).

Como foi dito anteriormente, no contexto moçambicano ainda há carência de material didático para o uso em sala de aula (AFRIMAP, 2012). Sendo assim, o livro didático configura-se como o único material que os professores utilizam para o planejamento das aulas. Desse modo, o livro virou o principal recurso para dar suas aulas (MACHADO, 1996). É na base desse material que o professor busca exemplos para o ensino, extrai exercícios, elabora os testes. E ainda com base nestas atividades organiza como irá comunicar os saberes aos alunos. 
Neste sentido, "o professor abdica do privilégio de projetar os caminhos a serem trilhados" para obedecer passivamente as orientações dos livros didáticos (Machado, 1996, p.31). Seguir de forma passiva as orientações dos livros didáticos pode prejudicar de forma grosseira a liberdade intelectual dos alunos pelo entendimento que os mesmos ao proceder comentários das atividades propostas, das atividades resolvidas, das ilustrações nele contidas bem como de outros aspectos incluídos neste documento podem trazer uma reflexão aos visados. Essa reflexão pode contribuir para que os alunos aprendam os conteúdos de forma relacional, isto é, adquiram uma compreensão em que no futuro consigam utilizar esses saberes em outros contextos.

Como forma de contornar a passividade dos alunos e do professor no uso de livro didático, o programa da $10^{a}$ classe indica que sejam realizadas atividades de medir; construir; usar tabelas; relacionar ângulos e distâncias; resolver problemas concretos relacionados com o cálculo de distâncias inacessíveis; determinar a altura de uma torre; determinar a largura de um rio. Nesse sentido "sugere-se que o aluno realize um trabalho de pesquisa sobre a história da trigonometria, a sua ligação com a vida e no âmbito da interdisciplinaridade" (INDE, 2010, p. 45).

\section{Teoria Antropológica do Didáctico}

Essa teoria é importante visto que visa produzir conhecimentos e o modo como esses conhecimentos devem ser ensinados para que os alunos os compreendam. Na sua essência, essa teoria baseia-se em duas praxeologias: a praxeologia matemática e a didática. Nesta seção, apresentamos a discussão dessa teórica como instrumento de análise de livros didáticos. É discutido o quarteto que a compõe e como pode ser usada para essa finalidade.

A Teoria Antropológica do Didático (TAD) surgiu a partir do projeto de investigação da Didática da Matemática levado a cabo na França na década 80. O francês Yves Chevallard é o pioneiro desta teoria (CHICOTE e DEIXA, 2016).

A TAD aparece como resposta ao problema de classificação de objetos matemáticos presente na Teoria de Transposição Didática (CHICOTE e DEIXA, 2016). A Transposição Didática é o processo de transformação adaptativa de um saber de uma instituição (BITTAR, 2017). A autora menciona que, segundo Chevallard, o saber não existe no vácuo.

Um dos constructos teóricos fundamentais da Teoria Antropológica do Didático é a noção de praxeologia. A palavra praxeologia provém de dois radicais: "praxis que significa prática, e logos, que significa estudo" (SANTOS e FREITAS, 2017, p. 52). Praxeologia é uma unidade básica que pode analisar a atividade humana em geral (MARTINEZ apud CHICOTE e DEIXA, 2016). Nessa perspectiva, a atividade humana em causa refere-se a matemática. Assim, podemos evidenciar dois tipos de praxeologias: praxeologias matemáticas (Organização Matemática) e praxeologia didática (Organização Didáctica) (BITTAR, 2017). 
A praxeologia matemática está ligada ao saber-fazer no sentido de produzir conhecimentos. Já a praxeologia didática se expõe ao fazer no sentido de agir, de ensinar alguém para que possa compreender e relacionar com outros saberes (CHEVALLARD, 2001). Na perspectiva desse autor, os objetos ostensivos e não ostensivos fazem parte das praxeologias e que estes objetos ostensivos se encontram articulados à tarefa e à técnica usada, ou seja, a linguagens da praxeologia. Essa linguagem pode ser escritural, figural, gráfica, verbal, gestual entre outras.

Chevallard (1999) refere que a TAD é organizada por dois blocos: prático-técnico e o tecnológico-teórico. O primeiro é formado pelos tipos de tarefas $(T)$ e as técnicas $(\tau)$ associadas a essas tarefas. $O$ segundo bloco é organizado pelas tecnologias $(\theta)$ e as teorias $(\Theta)$ nelas articuladas. O bloco prático-técnico apresenta as técnicas associadas para a determinação da solução da tarefa. Uma praxeologia relativa à tarefa T precisa de um modo de a fazer, portanto, de uma maneira de manipular uma certa tarefa. O bloco tecnológicoteórico expõe as justificações das técnicas utilizadas para a execução de um certo tipo de tarefa. Assim, uma tecnologia é um discurso racional que visa particularmente legitimar a técnica utilizada de modo que estejam claras as razões do recurso de tal técnica, de modo que ela possibilite a execução de tarefas do tipo $T$.

Em síntese, a TAD assume que qualquer atividade humana se baseia em resolver uma tarefa t de certo tipo $T$, por meio de uma técnica $\tau$, explicada por uma tecnologia $\theta$ que possibilita juntamente pensá-la, produzi-la. E a tecnologia é explicada por uma teoria $\Theta$. Desse modo, a TAD baseia-se na condição que toda a atividade humana se desdobra em uma organização praxeologica, designada por $[T, \tau, \theta, \Theta]$ (CHEVALLARD, 1999).

Esse elemento estrutural permite construir ou desconstruir uma atividade matemática. Por construir, nos referimos a possibilidade de elaborar um livro de Matemática onde o seu conteúdo está alicerçado nos pressupostos da TAD. Outra forma de construção tem a ver com a parte funcional da teoria: a praxeologia didática. Um professor pode planejar a sua ação na sala de aula, buscando pressupostos da TAD (SANTOS e FREITAS, 2017).

Por desconstruir, nos referimos a possibilidade de modelar uma organização matemática ou didática por meio dos elementos estruturais. Esse movimento permite extrair as estratégias empregadas na construção da organização matemática ou didática (CHICOTE e DEIXA, 2016). Observamos que esta é a vertente empregada em muitas pesquisas da área de Educação Matemática.

\section{Procedimentos metodológicos}

O estudo da trigonometria nos livros didáticos de Matemática do Sistema Nacional da Educação (SNE) Moçambicano foi executado considerando as tarefas não resolvidas. O objetivo deste estudo é analisar como o conteúdo matemático é abordado nos livros didáticos e em função das constatações sugerir técnicas que podem ser empregadas na 
realização de tarefas assim como na articulação do bloco tecnológico - teórico que justifique a técnica.

Para a concretização da análise foi necessária uma leitura dos Programas de Ensino da Matemática para verificar o que o Ministério da Educação e Desenvolvimento Humano (MINEDH) sugere para esse conteúdo matemático, sua localização em trimestre e quantidade de aulas propostas.

Em seguida, fez-se uma leitura dos livros. Essa leitura enquadra-se na análise de conteúdo proposta por Bardin (2012) que se fundamenta excessivamente nas suas três fases: a primeira fase - a pré-análise é a fase em que se organiza o material a ser analisado com o objetivo de torná-lo operacional. Para esta pesquisa foram identificados e organizados os seguintes documentos L1; L2; L3 e L4, cuja caracterização se encontra no quadro abaixo.

Nesta fase, estabeleceu-se o contato com o corpus (documentos analisados) e a determinação de indicadores por meio de recortes ou demarcação de texto nos documentos de análise. Salientamos que o material analisado nesta pesquisa faz parte de vários livros em uso no Ensino Secundário Geral (ESG). Sendo que os livros analisados foram de escolha intencional e a intenção deve-se a dois motivos: manuais aprovados pelo Ministério de tutela e, manuais publicados de acordo com o novo programa de Ensino em vigor no Sistema Nacional de Educação (SNE).

Quadro1: caracterização dos Livros analisados

\begin{tabular}{|c|c|c|c|c|}
\hline Livro & Autor & Titulo & Editora & Ano \\
\hline L1 & $\begin{array}{l}\text { Raul F. Carvalho \& Zeferino } \\
\text { A. Martins }\end{array}$ & Matemática 9a classe & Texto editores, Lda & 1999 \\
\hline L2 & Ismael C. Nhenze & Matemática $10^{a}$ Classe & Plural editores & 2017 \\
\hline L3 & $\begin{array}{l}\text { José P. Vuma\& Marcos } \\
\text { Cherinda }\end{array}$ & Matemática 11 & $\begin{array}{l}\text { Longman } \\
\text { Moçambique }\end{array}$ & 2009 \\
\hline L4 & $\begin{array}{l}\text { Arone Chilaule\& } \\
\text { Orlando Machango }\end{array}$ & Matemática $12^{a}$ classe & Texto editores, Lda & 2013 \\
\hline
\end{tabular}

A segunda fase-exploração do material que consiste na análise do material selecionado e organizado na fase anterior. Nessa fase, procede-se a identificação das unidades de registro e significação. Essas unidades correspondem ao segmento do conteúdo alvo, a trigonometria.

Dando prosseguimento, com base na análise preliminar dos quatro livros (L1; L2; L3 e L4) e definidos os tipos de tarefas T, contabilizadas as tarefas de cada tipo importa, desde já, realçar que pela teoria usada na análise desse material pretende-se perceber na avaliação do tipo de tarefa, se ela está bem identificada, se sua razão de ser está explicitada, se ela é adequada para alunos a que se destina; se o conjunto de tarefas fornece uma visão das situações matemáticas mais utilizadas. Desse modo, com finalidade de tornar os dados mais compressíveis adaptamos de Freitas \& Almoulound (2016) as seguintes unidades de registros: 
U1: Tarefas que exigem habilidades de leitura e interpretação; U2: Tarefas que exigem habilidades de cálculo com recurso a propriedades, regras e fórmulas e, U3: Tarefas que exigem a manipulação de registros.

A partir dessas unidades, fizemos uma releitura em paralelo com a teoria destacada, a $T A D$, salientar que esta fase é bastante importante porque possibilita ou não a riqueza das conclusões, isto é, com base na releitura e confrontação dos dados produzidos com a teoria de base, a TAD, pode-se compreender as articulações entre tarefas e técnicas usadas nesta unidade temática.

A terceira fase - tratamento dos resultados, inferência e interpretação, esta fase é destinada ao tratamento dos resultados; ocorre nela a condensação e o destaque das informações para análise, culminando nas interpretações inferenciais; é o momento da intuição, da análise reflexiva e crítica. (BARDIN, 2012).

\section{Apresentação e discussão dos dados}

Nesta seção, concentramos a nossa atenção para a apresentação, análise e discussão dos dados à luz da TAD. Primeiro apresentamos a análise e discussão dos Programas de Ensino de Matemática buscando compreender as orientações didáticas para o ensino desse conteúdo matemático no Ensino Secundário Geral e, em seguida, analisamos os livros didáticos. Na análise desses livros, buscamos compreender como o conteúdo é abordado considerando os tipos de tarefas, tipos de técnicas, tecnologias e as teorias mobilizadas.

Programa de Ensino de Matemática

De acordo com INDE (2010a), para a introdução da trigonometria os alunos deverão ser orientados a resolver atividades que conduzam a construção desse conceito com base na semelhança de triângulos. Nesse sentido, são recomendadas atividades como: medição, construção, uso de tabelas, relação entre ângulos e distâncias, realização de um trabalho de pesquisa sobre a história da trigonometria e sua ligação com a vida. Sugere ainda que os alunos resolvam problemas concretos ligados ao cálculo de distâncias inacessíveis; cálculo de altura de uma torre; largura de um rio, entre outras.

Em conformidade com as competências adquiridas no Ensino Secundário Geral do $1^{0}$ ciclo (ESG - 1), os programas do $2^{\circ}$ ciclo do ESG propõem que a trigonometria seja dada com base em diferentes problemas ligados às situações concretas, que permitam recordar e aplicar métodos trigonométricos. Nessa conformidade, os alunos devem compreender a importância do estudo da Trigonometria para as várias ciências, fazendo uso desta na sua vida cotidiano (INDE, 2010b; INDE, 2010c). Nesse ciclo, pretende-se que os alunos aprendam os conceitos de função periódica e de funções trigonométricas como modelos matemáticos adequados para responder a problemas do dia-a-dia dos alunos. 


\section{Análise de livros didáticos}

Conforme referenciamos nos procedimentos metodológicos, apresentamos o quadro que mostra a quantidade de tarefas para cada tipo de tarefa $T$ encontradas em cada livro analisado.

Quadro2: tipos de tarefas T e quantidade de tarefa identificados nos quatro livros analisados L1, L2, L3 e L4.

\begin{tabular}{|c|c|c|c|c|c|}
\hline T (Tarefa) & Descrição do Tipos de Tarefas & L1 & $\mathrm{L} 2$ & L3 & L4 \\
\hline $\mathrm{T}_{1}$ & Calcular as razões trigonométricas de um triângulo rectângulo. & 0 & 5 & 0 & 0 \\
\hline $\mathrm{T}_{2}$ & $\begin{array}{l}\text { Determinar um ângulo agudo conhecida uma das razões } \\
\text { trigonométricas. }\end{array}$ & 0 & 0 & 0 & 0 \\
\hline$T_{3}$ & Determinar as razões trigonométricas de um ângulo especial. & 6 & 0 & 0 & 0 \\
\hline $\mathrm{T}_{4}$ & $\begin{array}{l}\text { Determinar as razões trigonométricas aplicando a relação } \\
\text { fundamental entre seno e co-seno de um ângulo. }\end{array}$ & 0 & 3 & 2 & 0 \\
\hline$T_{5}$ & Resolver (in) equações trigonométricas simples. & 0 & 5 & 37 & 0 \\
\hline$T_{6}$ & Determinar os elementos de um triângulo rectângulo. & 0 & 0 & 0 & 0 \\
\hline $\mathrm{T}_{7}$ & Construir o gráfico de uma função trigonometria. & 0 & 2 & 3 & 3 \\
\hline $\mathrm{T}_{8}$ & Resolver os problemas práticos associados a vida real. & 0 & 0 & 0 & 0 \\
\hline$T_{9}$ & Representar um ângulo qualquer num círculo trigonométrico. & 0 & 3 & 0 & 0 \\
\hline $\mathrm{T}_{10}$ & $\begin{array}{l}\text { Converter a medida de um ângulo de graus em radianos e vice- } \\
\text { versa. }\end{array}$ & 0 & 2 & 0 & 6 \\
\hline $\mathrm{T}_{11}$ & $\begin{array}{l}\text { Aplicar as relações de complementaridade na obtenção das } \\
\text { razões trigonométricas de ângulos complementares. }\end{array}$ & 1 & 0 & 0 & 0 \\
\hline$T_{12}$ & $\begin{array}{l}\text { Determinar o período de uma função trigonometria, domínio e } \\
\text { contradomínio, equação de min e máx, ordenadas na origem. }\end{array}$ & 0 & 0 & 12 & 2 \\
\hline$T_{13}$ & Representar graficamente uma função trigonométrica. & 0 & 2 & 2 & 4 \\
\hline $\mathrm{T}_{14}$ & Fazer a leitura gráfica de funções trigonométricas. & 0 & 0 & 0 & 0 \\
\hline$T_{15}$ & $\begin{array}{l}\text { Determinar as razões trigonométricas de ângulos a partir do } \\
\text { círculo trigonométrico. }\end{array}$ & 0 & 5 & 0 & 0 \\
\hline$T_{16}$ & Determinara medida do ângulo e a amplitude & 0 & 0 & 7 & 0 \\
\hline$T_{17}$ & Determinar a área de uma figura & 1 & 0 & 2 & 0 \\
\hline
\end{tabular}

Apresentado o quadro acima e com base na unitarização feita e mostrada nos procedimentos metodológicos, contamos, analisamos e agrupamos as tarefas de acordo com as unidades de registros a que pertencem. Eis o quadro ilustrativo dos resultados da análise.

Quadro3: Quantidade de tarefas encontradas em cada livro para cada unidade de registro.

\begin{tabular}{c|c|c|c|c|c}
\hline Unidades de registros/ Livros & L1 & L2 & L3 & L4 & Total \\
\hline U1 & 0 & 12 & 5 & 0 & 17 \\
\hline U2 & 8 & 37 & 27 & 8 & 80 \\
\hline U3 & 0 & 12 & 7 & 7 & 26 \\
\hline Total & 8 & 61 & 33 & 15 & 123 \\
\hline
\end{tabular}

Os dados do quadro 3 mostram que os livros dão mais importância as tarefas do gênero calcular com uso de propriedades (80) em detrimento das tarefas do gênero que exigem a leitura e interpretação (17) bem como a de manipulação de registros (26). 
Lembrando ainda que pela teoria usada, a nossa análise se estende às técnicas, às tecnologias e às teorias. Na avaliação da técnica, pretendemos saber se ela é disponibilizada nos livros; na avaliação do bloco tecnologia-teoria, se está disponível nos livros e em casos afirmativos, como são dadas as justificativas tecnológicas. $\bigcirc$ quadro abaixo apresenta a sistematização da praxeologia, concretamente, os tipos de tarefa T e as técnicas presentes nesses livros.

Quadro 4: Síntese da praxe empregada nos livros analisados

\begin{tabular}{l|l}
\hline$T$ (tipo de tarefa) & $T$ (técnica) \\
\hline$T_{1}$ & Identificação dos catetos \\
\hline$T_{2,} T_{3}$ e $T_{16}$ & Uso de tabela trigonométrica de ângulos notáveis \\
\hline$T_{4}$ & Uso da relação fundamental da trigonometria \\
\hline$T_{5}$ e $T_{14}$ & Circulo trigonométrico e ou principio de equivalência \\
\hline$T_{6}$ e $T_{11}$ e $T_{17}$ & Aplicar as de complementaridade e as leis de seno e co-seno \\
\hline$T_{7} T_{12}$ e $T_{13}$ & $\begin{array}{l}\text { Leitura do círculo trigonométrico e respectivas translações em casos } \\
\text { de funções complexas }\end{array}$ \\
\hline$T_{8}$ & Leitura e contextualização \\
\hline$T_{9}$ & Redução de ângulos \\
\hline$T_{10}$ & Produto dos meios igual aos dos extremos \\
\hline$T_{15}$ & Apresentar e fazer a leitura no círculo trigonométrico \\
\hline
\end{tabular}

Articulação da tecnologia e teoria nos livros didáticos analisados

Para $T_{1}$ que é resolvida pela técnica $\tau_{1}$ : identificação dos catetos no triângulo rectângulo, encontra sua justificação tecnológica nas relações entre ângulos e lados em um triângulo rectângulo sendo esta a $\boldsymbol{\theta}_{1}$ que por sua vez encontra bases de sua sustentabilidade nas operações com triângulo que é para este caso $\Theta_{1}$, pois, nela encontramos as justificações ao alto nível para as razões que nos levam a usar esta técnica.

Para os tipos de tarefa $T_{2}, T_{3}$ e $T_{16}$ que são resolvidas pela técnica $\tau_{2}$ : Uso de tabela trigonométrica de ângulos notáveis, é justificada tecnologicamente pelo Teorema da soma dos ângulos internos de um triângulo sendo esta a $\boldsymbol{\theta}_{2} \mathrm{e}$ as operações com triângulo também justificam esta técnica, desta feita, encontramos que a $\Theta_{1}$ também é valida nessas tarefas.

$\mathrm{T}_{4}$ corresponde para este tipo de tarefa a técnica $\tau_{3}$ :descrita pelo uso da relação fundamental da trigonometria e justificada pela tecnologia $\boldsymbol{\theta}_{3}$ : descrita pelas propriedades do Teorema de Pitágoras que por sua vez é justificado ao alto nível pela teoria $\Theta_{1}$ operações com triângulo coincidentemente.

$\mathrm{T}_{5}$ e $\mathrm{T}_{14}$ resolvidas pela técnica $\tau_{4}$ : Círculo trigonométrico e ou princípio de equivalência que por sua vez são justificadas pela teoria $\boldsymbol{\bullet}_{\mathbf{4}}$ descrita na aplicabilidade do círculo trigonométrico por apresentar amplitudes e as razões trigonométricas e esta, por sua vez, 
justificada pela teoria $\Theta_{2}$ : :lescrita pelas importantes propriedades da Circunferência de raio unitário.

$T_{6}$ e $T_{11}$ e $T_{17}$ para esse conjunto de tipos de tarefa, contam com a aplicação das relações de complementaridade e as leis de seno e co-seno como técnica sendo esta a $\boldsymbol{\tau}_{\mathbf{5}}$ que encontra a base nos seguintes enunciados: em qualquer triângulo a medida dos lados é proporcional aos seus lados opostos e em qualquer triângulo, o quadrado da medida de um lado é igual à soma dos quadrados das medidas dos outros lados menos o dobro do produto das medidas desses lados pelo co-seno do ângulo formado por eles. Sendo esta a $\boldsymbol{\Theta}_{5}$ que é justificada pela teoria $\Theta_{1}$ operações com triângulos.

$\mathrm{T}_{7}$ e $\mathrm{T}_{12}$ e $\mathrm{T}_{13}$ para este conjunto, a leitura do círculo trigonométrico e as respectivas translações, em casos de funções complexas, é a técnica: $\boldsymbol{\tau}_{5}$ para as tarefas e esta por sua vez encontra suas bases fundamentadas nas relação existente entre os elementos de um triângulo (ângulos e lados) que é $\mathbf{\theta}_{6}$ justificada pela teoria $\Theta_{1}$.

$\mathrm{T}_{8}$ para esse tipo de tarefa encontra como técnica a leitura e contextualização $\tau_{6} \mathrm{e}$ a tecnologia para este caso é a circunferência unitárias e translação de gráficos nos eixos cartesianos $\boldsymbol{\theta}_{7}$ para teoria encontramos $\Theta_{3}$ funções reais de variáveis reais.

$T_{9}$ - a técnica e descrita em termos de redução de ângulos: $\boldsymbol{x}_{7}$ e a divisão entre a amplitude dada, pela amplitude de uma volta e a tecnologia $\boldsymbol{\bullet}_{g}$ justificada pelo teorema da divisão $\Theta_{4}$. $T_{10}$ - encontra como técnica $O$ produto dos meios é igual ao produto dos extremos $\tau_{\mathbf{8}} \mathrm{e}$ a correspondência entre as amplitudes e suas respectivas razões trigonométricas $\theta_{7}$ e como teoria encontramos teorema da divisão $\Theta_{3}$. $T_{15}$ - apresentar e fazer a leitura no círculo trigonométrico é a técnica que se pode usar para tarefas deste tipo sendo esta a $\boldsymbol{\theta}_{8}$ e a teoria que se enquadra nestas tarefas é $\Theta_{2}$ :descrita pelas importantes propriedades da circunferência de raio unitário.

\section{Considerações finais}

Da análise das tarefas propostas nos quatro livros didáticos percebemos que as técnicas, tecnologias e teorias apresentadas são em grande parte a produção dos autores tentando mostrar a melhor estrutura que o ensino de um conteúdo pode apresentar. Os resultados da pesquisa nos levam a afirmar que os livros analisados não cumprem as sugestões dos programas de Ensino da Matemática. Constatamos que há predominância de tarefas do gênero calcular com uso de propriedades, sendo 80 das 123 tarefas estão associadas a este gênero. Para a resolução dessas tarefas, o aluno necessita somente conhecer e saber aplicar as fórmulas e algoritmos e propriedades. Verificamos ainda que 26 das 123 exigem habilidades de manipulação dos registros e, 17 das 123 tarefas exigem a leitura e interpretação. 
Ao confrontarmos esses dados com os Programas de Ensino de Matemática, percebemos que há uma desconexão entre as tarefas dos quatro livros e as sugestões dos programas de ensino. Os Programas de Matemática do ESG sugerem que o ensino da trigonometria seja feito com uso de situações - problemas e tarefas que contribuem para a construção do conhecimento, ou seja, tarefas que permitem ao aluno vivenciar uma Matemática que se usa na vida. A realidade indicou que os livros possuem muitas tarefas que conduzem a reprodução em detrimento das que levam a construção de conhecimentos pelos alunos. Perante essa ocorrência sugerimos que na realização da atividade docente, além do uso do livro didático, o professor também possa fazer o uso dos programas de ensino de forma a visualizar o que é recomendado e, assim, conclui-se haver a necessidade de leitura de mais de um livro para a preparação e o planejamento de aulas.

A predominância de tarefas do género calcular pode explicar as razões que levam os alunos a considerar a trigonometria como sendo difícil visto que nessa unidade o aluno se depara com muitas fórmulas e propriedades que são compreendidas de forma mecânica.

\section{Referências}

AFRIMAP. A prestação efectiva de serviços Públicos no sector da educação em Moçambique. 2012.

BARDIN, L. Análise de Conteúdo. Lisboa, Portugal; Edições 70, LDA, 2012.

BITTAR, M. A teoria Antropológica do Didáctico como ferramenta metodológica para a análise de livros didacticos. Zetetiké, Campinas, SP. v.25.n.3, p. 364-387, set/dez. 2017.

CHEVALLARD, Y. Conceitos Fundamentais da Didáctica: as perspectivas trazidas por uma abordagem antropológica. In: BRUM, Jean (Org.). Didáctica das matemáticas. Lisboa: Instituto Piaget, 1996.

CHEVALLARD, Y.; BOSCH, M. e GASCÓN, J. Estudar Matemáticas: o elo perdido entre o ensino e a aprendizagem. Porto Alegre, Artmed Editora, 2001.

CHICOTE, R. S.; DEIXA, G. Análise de tarefas em Livros escolares da $11^{\text {a }}$ classe utilizados em Moçambique na perspectiva da Teoria Antropológica do Didáctico. KULAMBELA: Revista Moçambicana de Ciências e Estudos da Educação. Cabo Delgado, Moçambique, vol.03. n.09. p. 46-57 março, 2016.

FREITAS, R. L.; ALMOULOUD, S. A: Análise de livro didáctico e a construção de um processo de ensino por meio de tarefas e técnicas: contribuições da TAD. In: Jesus Flores Salazar \& Francisco Ugarte Guerra (Orgs.). Investigaciones En Educacion Matematica: Fondo editorial, 2016, P. 217-237.

INDE. Matemática, Programa da 10ª Classe. DINAME, Maputo, 2010a.

INDE. Matemática, Programa da $11^{\mathrm{a}}$ Classe. DINAME, Maputo, 2010b.

INDE. Matemática, Programa da $12^{a}$ Classe. DINAME, Maputo, 2010c. 
MACHADO, N. J. Sobre livros didácticos: quatro pontos. Em Aberto, Brasília, v. 26, n. 69, pp.22-27, jan./mar. 1996.

SANTOS, C. M. dos., FREITAS, J. L. M. de. Contribuições da teoria antropológica do didáctico na formação de professor de matemática. Amazônia, Revista de Educação em Ciências e Matemática | v.13 (27). p.51-66. Set 2017.

PONTE, J. P. Gestão curricular em Matemática. In GTI (Ed.), O professor e o desenvolvimento curricular (pp. 11-34). Lisboa: APM, 2006. 